\title{
Noncontact Screening Methods for the Detection of Narrow Anterior Chamber Angles
}

\author{
Priya L. Dabasia, ${ }^{1}$ David F. Edgar, ${ }^{1}$ Ian E. Murdoch, ${ }^{2}$ and John G. Lawrenson ${ }^{1}$ \\ ${ }^{1}$ City University London, London, United Kingdom \\ ${ }^{2}$ UCL Institute of Ophthalmology, London, United Kingdom
}

Correspondence: John G. Lawrenson, Division of Optometry and Visual Science, City University London, Northampton Square, EC1V OHB, UK;

J.G.Lawrenson@city.ac.uk.

Submitted: February 22, 2015

Accepted: May 5, 2015

Citation: Dabasia PL, Edgar DF, Murdoch IE, Lawrenson JG. Noncontact screening methods for the detection of narrow anterior chamber angles. Invest Ophthalmol Vis Sci. 2015;56:3929-3935. DOI:10.1167/ iovs. $15-16727$
Purpose. Comparing diagnostic accuracy of biomicroscope techniques (van Herick and Smith's tests, evaluating limbal and central anterior chamber depth, respectively) and advanced imaging (Visante OCT and Pentacam) for detection of gonioscopically narrow anterior chamber angles (ACAs).

Methods. A total of 78 subjects with narrow or open ACAs underwent four index tests, performed on both eyes by examiners masked to other test results. Diagnostic performance was compared with gonioscopy, using International Society of Geographical and Epidemiological Ophthalmology (ISGEO) definition of primary angle closure and a classification based on clinical opinion of occludability. Data were analyzed using both the eye and the individual as unit of analysis. Sensitivity, specificity, and partial area under the receiver operating characteristic curve (AUROC) were generated.

Results. Using the eye as the unit of analysis, the van Herick grading cutoff of $25 \%$ or less and ISGEO gonioscopic classification achieved $80 \%$ (confidence interval [CI] 65 to 89) sensitivity and $92 \%$ specificity (CI 80 to 97) for narrow angle detection, with specificity reaching $97 \%$ (CI 87 to 100 ) for a cutoff of less than or equal to $15 \%$. Notably, with a gonioscopic classification based on clinical opinion of occludability, van Herick $(\leq 25 \%)$ together with Smith's test $(\leq 2.50 \mathrm{~mm})$ detected $100 \%$ of narrow angle subjects. Of the three Pentacam parameters, anterior chamber volume achieved highest test sensitivity of $85 \%$ (CI 70 to 94 ) using the ISGEO definition. Visante OCT ACA had greatest partial AUROC at 90\% specificity, also yielding sensitivity and specificity greater than $85 \%$ using the Youden-derived cutoff of less than or equal to $20.7^{\circ}$ and ISGEO definition.

Concuusions. Van Herick test and Visante OCT ACA exhibited best discrimination between narrow and open angles both alone, and in combination. Van Herick test affords advantages over Visante OCT, showing potential for identifying individuals who may benefit from further gonioscopic assessment in a case-finding or screening setting.

Keywords: gonioscopy, van Herick test, advanced anterior segment imaging, diagnostic accuracy, angle closure glaucoma

\begin{abstract}
A ngle closure glaucoma (ACG) is a major cause of visual morbidity. With the aging population and increasing longevity, the World Health Organization estimates that of the 11.2 million people who will be bilaterally blind from glaucoma worldwide by 2020 , nearly half will be attributed to angle closure mechanisms. ${ }^{1}$

Prevention of ACG through screening depends on timely identification of individuals with anatomically narrow angles, considered at risk of developing the condition. Currently, gonioscopy is considered the reference-standard assessment for anterior chamber angle (ACA) configuration. However, the technique requires considerable skill and experience. Therefore, this clinical reference-standard technique is considered unsuitable for case-finding or large-scale population screening. Surrogate methods to assess ACA configuration include biomicroscope-based techniques, such as the van Herick ${ }^{2}$ and/ or Smith's tests, ${ }^{3}$ which evaluate limbal anterior chamber depth (LACD) and central ACD, respectively. Newer advanced optical-based systems provide noncontact, objective, and quantifiable methods for evaluating the ACA, acquiring data
\end{abstract}

Copyright 2015 The Association for Research in Vision and Ophthalmology, Inc. iovs.arvojournals.org | ISSN: 1552-5783 rapidly, which can be easily stored. Both anterior-segment optical coherence tomography (AS-OCT) ${ }^{4-10}$ and Pentacam imaging $^{10-14}$ have been used to image ACA structures and generate quantitative estimates of angle morphology for use in screening for ACG in at-risk populations.

Most research into the effectiveness of biomicroscope tests and optical imaging-based systems to detect angle closure disease has been undertaken in East Asia, where prevalence and mechanisms of angle closure differ significantly from other populations. ${ }^{15}$ The present study aimed to evaluate diagnostic accuracy of noncontact methods in screening for narrow angles, compared with gonioscopy, in an enriched population. The study was designed, and findings reported in accordance with the Standards for Reporting of Diagnostic Accuracy criteria. ${ }^{16}$

\section{MeTHODS}

Data collection for this prospective, diagnostic accuracy study took place in Ealing Hospital, Moorfields Eye Clinic, in 2014. 
The study had institutional review board approval and was conducted in accordance with the Declaration of Helsinki. Written informed consent was obtained from all subjects.

Adult subjects were recruited from glaucoma and general ophthalmology clinics. The narrow angle group comprised subjects with suspected and confirmed primary angle closure. The open angle group had no current or previous history of ocular disease, or were diagnosed with eye conditions not affecting angle configuration. Subjects receiving systemic or topical medications known to affect the anterior segment, and in particular those that may influence ACA configuration (e.g., miotics), were excluded. Other exclusion criteria included anomalies of the anterior segment that affect ACA configuration. Phakic eyes from both groups were included for analysis.

Subjects underwent assessment using gonioscopy and index tests comprising van Herick, Smith's, and imaging using the Pentacam (OCULUS Optikgeräte GmbH, Wetzlar, Germany) and Visante AS-OCT (Carl Zeiss Meditec AG, Oberkochen, Germany) (on the same day), and without use of mydriatics. All tests were performed in uniform dark-room conditions confirmed using a digital photometer (ISO-TECH ILM 350 digital light meter; Isothermal Technology Limited, Southport, UK) as 5 lux or less at the level of the subject's eye.

Each index test was performed by a single experienced examiner without previous knowledge of the subjects' ocular status and masked to other test results, including gonioscopy.

\section{Van Herick Test}

Using methodology originally described by van Herick et al., ${ }^{2}$ the width of the corneal section was compared with the adjacent anterior chamber space, first at the temporal limbus and then at the nasal limbus for each eye, but recorded as a percentage in accordance with the modified 7-point grading scale $(0 \%, 5 \%, 15 \%, 25 \%, 40 \%, 75 \%$, and $\geq 100 \%)$ of Foster and colleagues. ${ }^{17}$

\section{Smith's Test}

Redmond Smith ${ }^{3}$ proposed a quantitative method to estimate central ACD using the calibrated variable slit-height facility on the biomicroscope. The examiner projects a horizontal slit across the central cornea, and adjusts the slit-height until the light beam from the corneal surface and lenticular-iridal interface just appear to touch (see $\mathrm{Smith}^{3}$ for further details on testing procedure). Smith's report ${ }^{3}$ proposed multiplying the slit height registered on the biomicroscope scale by a constant correction factor of 1.40 to determine the estimated ACD in millimeters. In our study, Smith's test results were calculated using the 1.31 correction factor suggested by Barrett et al., ${ }^{18}$ and based on a smaller mean bias determined by BlandAltman difference analysis when Smith's central ACD estimates were compared with the imaging systems (Visante OCT and Pentacam). Three readings were taken for each eye, resetting the slit height to $0.5 \mathrm{~mm}$ between measurements, and recording the mean result for analysis.

\section{Pentacam Imaging}

The Pentacam, incorporating software version 1.19r11 (OCULUS Optikgeräte $\mathrm{GmbH}$ ), was used in 25-image acquisition, and automatic release mode. The inbuilt Pentacam software generates three anterior chamber parameters: ACA, central ACD, and anterior chamber volume (ACV) (Pentacam imaging principles have been described elsewhere ${ }^{19}$ ). The ACA estimates were obtained along the nasal-temporal meridian using Scheimpflug horizontal image segment 16 (184 to $\left.4^{\circ}\right)$. Scan acquisition was repeated until two scans of suitable quality, or a maximum of four scans had been captured using manufacturer-recommended quality criteria for image acceptance.

\section{Visante AS-OCT}

The Visante OCT (Version 2.0.1.88; Carl Zeiss Meditec AG) was used in "anterior segment single" mode using wide-field scanning optics to obtain a cross-section of the nasal and temporal angles in a single, $16 \times 6 \mathrm{~mm}$ image frame between the 3 and 9 o'clock positions (see Penner ${ }^{20}$ for further details). All images were analyzed using the Visante OCT inbuilt software by one experienced clinician (PLD) masked to the gonioscopy findings and index test results. Angle tool markers (AC-angle- $180^{\circ}$ and $\mathrm{AC}$-angle- $0^{\circ}$ ) were positioned at the deepest points of the angle recess, adjusting the long arms of the tool at the iris tangential line and posterior corneal surface. Central ACD was measured, using the caliper tool selected from the "chamber tool palette," as the distance between the corneal apex in a line perpendicular to the posterior surface of the cornea (endothelium) and anterior lens contour.

\section{Gonioscopy}

Every subject underwent gonioscopy on the same day as the index tests, performed by the same consultant glaucoma subspecialist ophthalmologist with extensive experience in performing the technique, and previously standardized against another consultant ophthalmologist with a weighted kappa scoring of 0.88 (SE 0.07) for Shaffer angle grading. The ophthalmologist was masked to the subjects' ocular status and results of index tests. Angle width was estimated using the Modified Shaffer system. Dynamic assessment by further compression of the corneal surface by the goniolens was also performed to differentiate appositional closure from peripheral anterior synechiae. The examiner made a "forced" choice as to whether the angle of each eye was "occludable" (a narrow angle with possibility of occlusion) or "not occludable" (at little risk of occlusion) based on the following criteria:

1. Angular approach of the peripheral iris to the recess and peripheral iris configuration (e.g., steep)

2. Angle structures observed with the subject's eye in the primary position (modified Shaffer grading)

3. "Openability": visibility of angle structures on indentation

4. Observation of other features suggestive of iridotrabecular contact (e.g., pigment patches)

\section{Diagnostic Definitions}

As the primary aim of this study was to evaluate screening methods for the detection of narrow angles, subjects diagnosed as primary angle closure suspect, primary angle closure (PAC), and primary angle closure glaucoma (PACG) were combined into a single category: "narrow" or "occludable" angles. Using gonioscopy as the reference standard, an eye was defined as having a narrow or occludable angle using two criteria:

1. International Society of Geographical and Epidemiological Ophthalmology (ISGEO) definition, defined as an ACA in which the posterior (usually pigmented) trabecular meshwork was not visible for $270^{\circ}$ or more of the angular extent on nonindentation gonioscopy and with the eye in the primary position. ${ }^{11,17,21-23}$

2. Clinical opinion of the consultant subspecialist ophthalmologist as to whether the angle was "occludable." This "pragmatic" criterion provides a measure of the ability 
of the index tests to identify individuals who would be most likely to benefit from treatment.

\section{Sample Size Calculation}

The sample size was based on an anticipated sensitivity of the van Herick test to detect a narrow angle (gonioscopic definition) of 0.80 (conservative estimate from a study in a population of European descent ${ }^{24}$ ) with a minimum acceptable precision of \pm 0.25 with 0.95 probability. This would require 40 cases with narrow angles. ${ }^{25}$

\section{Statistical Analysis}

Statistical analysis was performed using SPSS 21.0 software (http://www-01.ibm.com/software/analytics/spss/products/ statistics/downloads.html), Medcalc 14.8.1 (www.medcalc. org), and STATA 13.0 (Stata Corp., College Station, TX; www. stata.com). Mean/median values for demographic characteristics and quantitative angle measurements were compared between gonioscopically narrow and open angle groups using parametric or non-parametric statistical tests as appropriate. For all tests $P<0.05$ was considered statistically significant.

The diagnostic effectiveness of the index tests was evaluated using two approaches:

- Primary analysis: using the eye as the unit of analysis and comparing the gonioscopy reference standard and index test results for the right eye. Left eye data were included for analysis only if the right eye was not eligible for inclusion in the study.

- Secondary analysis: using the individual as the unit of analysis and comparing the narrowest index test measurement of the two eyes with the narrowest gonioscopic classification recorded for each subject. These data are more generalizable to case-finding/screening for narrow/ occludable angles.

For index tests without a clinical consensus on the threshold to define a narrow angle, the optimal threshold was determined from the receiver operator characteristic (ROC) curve using the Youden index (J). The Youden index represents the point on the curve that maximizes $\mathrm{J}$ in the formula, $J=\max$ (sensitivity $[\mathrm{c}]+\operatorname{specificity}[\mathrm{c}]-1$ ), where $\mathrm{c}$ ranges over all possible criterion values. ${ }^{26}$ The ability to discriminate between narrow- and open-angle subjects for continuous data was described using partial area under the ROC (AUROC) curve estimates (normalized by dividing by the false-positive rate ${ }^{27}$ ), together with $95 \%$ confidence intervals (CIs). The diagnostic effectiveness of combining index test results was evaluated using $2 \times 2$ tables to calculate sensitivity and specificity based on failure of one or both index tests being suggestive of a narrow ACA.

\section{Results}

A total of 78 subjects, with both narrow and open ACAs (34 male and 44 female), attended one of two screening days. Demographic and summary data for open and narrow angle groups are summarized in Table 1, with a subject being classified as having gonioscopically narrow angles if either eye satisfied the criteria. Subjects were aged between 30 and 83 years with median age of 66 years (interquartile range [IQR] 53 to 79). Self-reported ethnicities were $56 \%$ white and $35 \%$ South Asian. Subjects classified with narrow angles were statistically significantly older $(P=0.008$, ISGEO classification; $P=0.046$, classification based on clinical opinion of occludability), and had higher IOPs $(P=0.038$, ISGEO classification; $P=0.009$, classification based on clinical opinion) than those in the open angle group. By defining a narrow angle as $\geq 270^{\circ}$ nonvisibility of the posterior trabecular meshwork (ISGEO classification), $46 \%(n=36)$ and $54 \%(n=42)$ of subjects were diagnosed with open and narrow ACAs, respectively. The percentage with narrow angles fell to $21.8 \%(n=17)$ if the clinical opinion of occludability was used as the cutoff criterion.

In our cohort, the biomicroscope-based tests (van Herick and Smith's) and reference comparison gonioscopic examination captured data of suitable quality for analysis in $100 \%$ of eyes $(n=145)$. Following repeat acquisition in accordance with the study protocol, the imaging-based systems (Visante OCT and Pentacam) acquired adequate-quality data for the measurement of ACA and ACD in $88 \%$ to $97 \%$, and $96 \%$ to $100 \%$ of eyes, respectively, with Pentacam nasal ACA being the parameter with the greatest proportion of data excluded from analysis (12\% for left eye data). No bias was observed between narrow and open angle groups for data excluded on the basis of poor quality.

For the primary analysis, the diagnostic performance of each index test was evaluated against two gonioscopy reference standards (ISGEO classification and clinician's judgment of occludability) and using the eye as the unit of analysis. The analysis was repeated using the individual as the unit of analysis, yielding similar results (data not shown).

\section{Diagnostic Effectiveness of Biomicroscope-Based Index Tests}

Using the traditional van Herick cutoff criterion of grade 2 (modified LACD $\leq 25 \%$ ) and by defining a narrow angle using the ISGEO gonioscopy classification, the van Herick test achieved $79.5 \%$ (CI 64.5-89.2) sensitivity and 92.3\% (CI 79.7-97.3) specificity. Figure 1 provides a graphical representation of test sensitivities and specificities using various cutoffs for best-performing biomicroscope and imaging tests (tabulated results are provided in Table 2 ). In comparison, a similarly high test sensitivity and specificity exceeding $80 \%$ was obtained at the $\leq 15 \%$ LACD cutoff when using the gonioscopic classification of an occludable angle based on clinical opinion. The Youden index-derived optimal cutoff for Smith's central ACD was $2.6 \mathrm{~mm}$ or less, based on the ISGEO classification of the angle, yielding lower test sensitivity and specificity of 71.8\% (CI 56.2-83.5) when compared with LACD observations (Youden cutoff $\leq 25 \%$ ). Subanalysis of the diagnostic effectiveness of nasal and temporal LACD revealed no statistically significant differences for sensitivity or specificity $(P=1.0$ McNemar test) using the $\leq 25 \%$ cutoff and ISGEO gonioscopic definition of a narrow angle.

\section{Diagnostic Effectiveness of Imaging-Based Index Tests}

Youden index-derived cutoffs for ACA were $20.7^{\circ}$ and $30.7^{\circ}$ using Visante OCT (Fig. 1) and Pentacam imaging, respectively, based on the ISGEO gonioscopy classification. Using these criteria, Visante OCT ACA showed better sensitivity and specificity (exceeding 85\%) than Pentacam-derived estimates (Table 2). Central ACD measurements generated by both devices showed similar sensitivities $(71.8 \%$, Visante OCT and $74.4 \%$, Pentacam) compared with Smith's test $(\leq 2.60 \mathrm{~mm})$, but higher specificities (84.6\%, Visante OCT and $76.3 \%$, Pentacam) at the $\leq 2.50 \mathrm{~mm}$ Youden cutoff. Of the three Pentacam anterior chamber parameters, ACV (Youden cutoff $\leq 124 \mathrm{~mm}^{3}$ ) achieved the highest test sensitivity of $84.6 \%$ (CI 69.5-94.1) using the ISGEO definition of a narrow angle. Further analysis of Visante OCT ACA data found similar effectiveness of temporal and nasal measurements to detect narrow angles, 
TABLE 1. Demographic and Clinical Data for Narrow and Open Angle Subject Groups Using Two Different Classifications of a Narrow ACA by Gonioscopy (a Subject Was Classified as Having Gonioscopically Narrow Angles if One or Both Eyes Satisfied the Diagnostic Criteria)

Classification of Narrow ACA by Gonioscopy

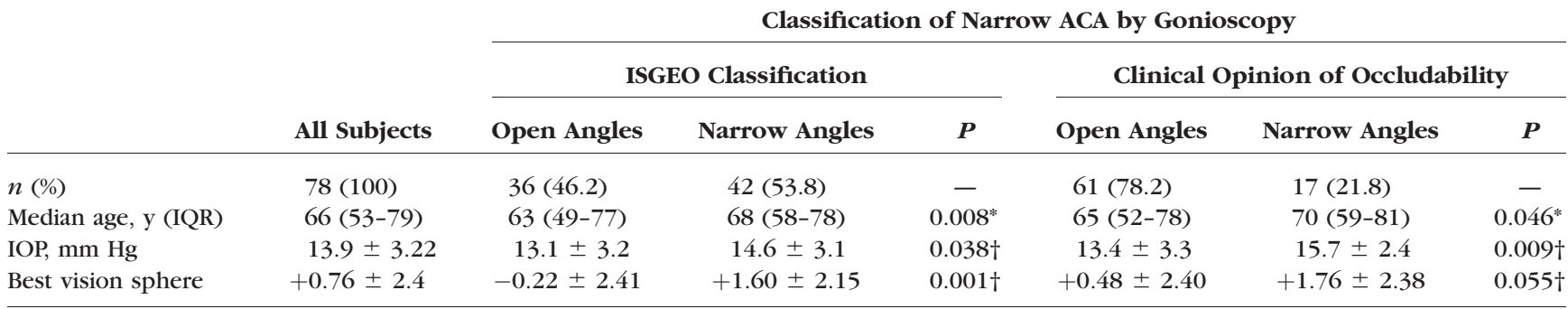

Comparisons between narrow and open ACA groups produced statistically significant differences $(P<0.05)$

* Mann-Whitney $U$ test.

† Independent-sample $t$-test.

with no statistically significant difference observed for either sensitivity or specificity ( $P=1.0 \mathrm{McNemar}$ test). Interestingly, Pentacam imaging showed a marked difference in diagnostic performance between temporal and nasal angle positions, with higher mean ACA measured at the temporal limbus compared with the nasal position. Bland-Altman mean difference analysis revealed mean bias of $2.37^{\circ}$ (CI -7.18 to 11.93 ).

Given the low prevalence of narrow angles in Western populations, a test specificity of $90 \%$ or greater is essential to screen for the condition. Table 2 details partial AUROC estimates at $90 \%$ and $95 \%$ specificity together with their $95 \%$ CIs. At $90 \%$ specificity and using the ISGEO definition of a narrow angle, the Visante ACA (0.63, CI 0.48-0.84) and van Herick test $(0.49$, CI $0.20-0.82)$ generated the greatest partial AUROCs. No significant difference was observed between the two parameters for partial AUROC curve estimates for ranges starting at $90 \%$ or $95 \%$ specificities using either gonioscopy classification $(P>0.14)$

\section{Combining Test Results}

Using the ISGEO gonioscopy classification system and based on failure of both the van Herick test $(\leq 25 \%)$ AND Smith's test $(\leq 2.60 \mathrm{~mm})$ being suggestive of a narrow angle, test specificity of $95 \%$ could be achieved but this was offset by a reduction in sensitivity below $60 \%$. Conversely, detection of a narrow angle based on failure of EITHER test using the same criteria improves sensitivity above $90 \%$, but reduces test specificity to just below $70 \%$. Combining the two biomicroscope-based tests is logical because the tests may be performed in rapid succession. However, based on failure of both best-performing index tests and using the ISGEO gonioscopy classification, the van Herick $(<25 \%)$ and Visante OCT ACA $\left(<20.7^{\circ}\right)$ elicit $97 \%$ specificity, while still retaining a sensitivity of $74 \%$. Notably, by using a gonioscopic classification based on clinical opinion of occludability, $100 \%$ of narrow angle subjects were detected using the van Herick technique $(\leq 25 \%)$ together with Smith's test $(\leq 2.50 \mathrm{~mm})$.

\section{DisCUSSION}

Although gonioscopy is considered to be the reference standard for the determination of ACA configuration, the technique is unsuitable for large-scale screening or case finding of individuals at risk of ACG. This study evaluated the diagnostic accuracy of alternative noninvasive methods to detect occludable angles. In our population, both biomicroscope-based tests (van Herick and Smith's) showed good diagnostic performance for the detection of gonioscopically narrow ACAs, comparable with modern anterior segment imaging systems (Pentacam and Visante OCT). Furthermore, these biomicroscopic techniques were quick to perform, were well accepted by patients, and could be readily incorporated into a standard ophthalmic examination.

Van Herick et al. ${ }^{2}$ indicated that an LACD of grade 2 or less (equating to $\leq 25 \%$ of the corneal thickness) may suggest a narrow ACA and these cases should be investigated further by gonioscopy. Using the eye as the unit of analysis, the current study confirmed that this cutoff provided the optimal balance between sensitivity and specificity for detection of a narrow angle, as defined by the ISGEO classification. Several studies have reported on the performance of the van Herick test for detection of gonioscopically occludable angles. $4,6,17,23,28,29$ Some have described its superior performance over other noninvasive screening tests, such as ultrasound pachymetry, optical pachymetry, or the scanning peripheral anterior chamber depth analyzer. ${ }^{23,29}$ Conversely, others questioned
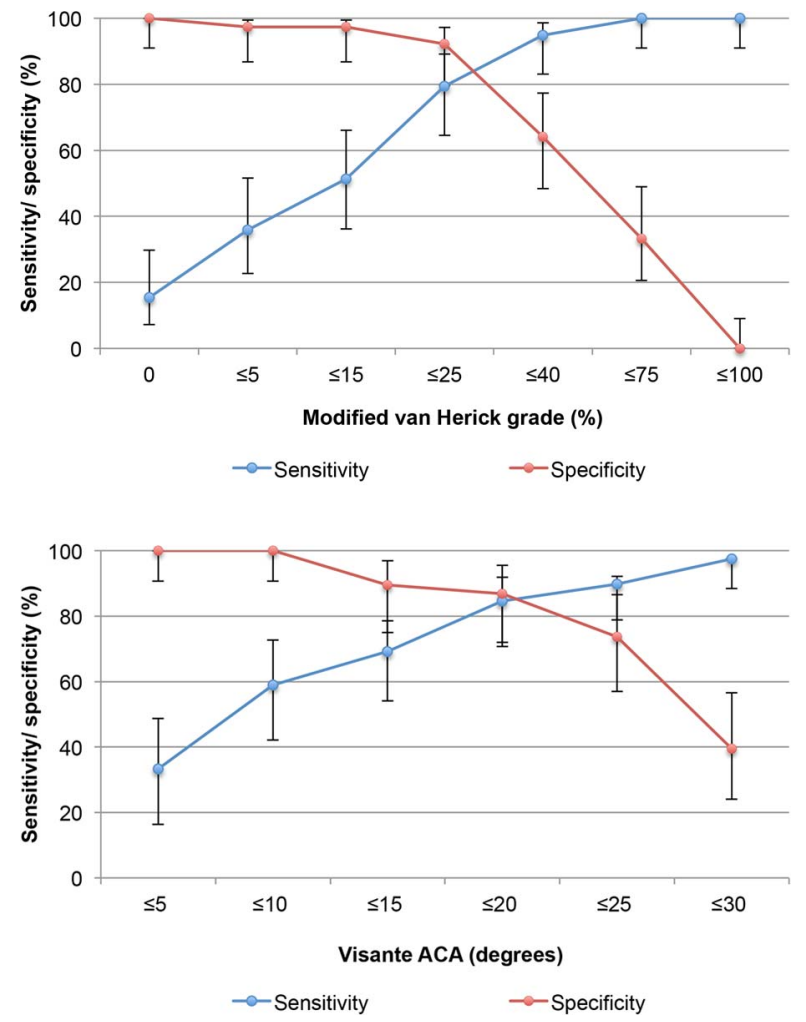

FiguRE 1. Sensitivity-specificity plots for best-performing biomicroscope and imaging tests, the van Herick technique and Visante OCT ACA, using the ISGEO gonioscopy classification and based on the eye as the unit of analysis. 
Table 2. Sensitivity, Specificity, and Partial AUROC Curve Data for Each Index Test Parameter Using the Two Gonioscopic Classifications for a Narrow Angle and Based on the Eye as the Unit of Analysis

\begin{tabular}{|c|c|c|c|c|c|c|}
\hline Index Test & $\begin{array}{c}\text { Index } \\
\text { Test Cutoff }\end{array}$ & $\begin{array}{c}\text { Reference } \\
\text { Standard Cutoff }\end{array}$ & $\begin{array}{l}\text { Sensitivity, } \\
\quad \% \text { (CI) }\end{array}$ & $\begin{array}{l}\text { Specificity } \\
\%(C I)\end{array}$ & $\begin{array}{c}\text { Partial } \\
\text { AUROC } \\
\text { From 90\% } \\
\text { Specificity (CI) }\end{array}$ & $\begin{array}{c}\text { Partial } \\
\text { AUROC } \\
\text { From 95\% } \\
\text { Specificity (CI) }\end{array}$ \\
\hline \multirow[t]{2}{*}{ van Herick } & $\leq 25 \%$ & ISGEO & $79.5(64.5-89.2)$ & $92.3(79.7-97.3)$ & $0.49(0.20-0.82)$ & $0.33(0.09-0.80)$ \\
\hline & & Clinical opinion & $94.1(73.0-99.0)$ & $70.5(58.1-80.4)$ & $0.30(0.08-0.70)$ & $0.20(0.0-0.54)$ \\
\hline \multirow[t]{2}{*}{ Smith's } & $\mathrm{ACD} \leq 2.60 \mathrm{~mm}$ & ISGEO & $71.8(56.2-83.5)$ & $71.8(56.2-83.5)$ & $0.29(0.14-0.53)$ & $0.24(0.09-0.47)$ \\
\hline & $\mathrm{ACD} \leq 2.50 \mathrm{~mm}$ & Clinical opinion & $76.5(52.7-90.4)$ & $70.5(58.1-80.4)$ & $0.19(0.02-0.51)$ & $0.06(0.0-0.40)$ \\
\hline \multirow[t]{4}{*}{ Visante OCT } & $\mathrm{ACA} \leq 20.7^{\circ}$ & ISGEO & $87.2(72.6-95.7)$ & $86.8(71.9-95.6)$ & $0.63(0.48-0.84)$ & $0.62(0.46-0.80)$ \\
\hline & $\mathrm{ACA} \leq 18.6^{\circ}$ & Clinical opinion & $100(80.5-100.0)$ & $66.7(53.3-78.3)$ & $0.31(0.07-0.67)$ & $0.16(0.0-0.59)$ \\
\hline & $\mathrm{ACD} \leq 2.50 \mathrm{~mm}$ & ISGEO & $71.8(55.1-85.0)$ & $84.6(69.5-94.1)$ & $0.40(0.20-0.69)$ & $0.30(0.13-0.65)$ \\
\hline & $\mathrm{ACD} \leq 2.38 \mathrm{~mm}$ & Clinical opinion & $82.3(56.6-96.2)$ & $83.6(71.9-91.8)$ & $0.38(0.13-0.70)$ & $0.24(0.0-0.63)$ \\
\hline \multirow[t]{6}{*}{ Pentacam } & $\mathrm{ACA} \leq 30.7^{\circ}$ & ISGEO & $71.8(55.1-85.0)$ & $78.4(61.8-90.2)$ & $0.37(0.16-0.61)$ & $0.25(0.10-0.58)$ \\
\hline & $\mathrm{ACA} \leq 30.2^{\circ}$ & Clinical opinion & $82.4(56.6-96.2)$ & $62.7(49.1-75.0)$ & $0.36(0.14-0.62)$ & $0.31(0.1-0.58)$ \\
\hline & $\mathrm{ACD} \leq 2.50 \mathrm{~mm}$ & ISGEO & $74.4(57.9-87.0)$ & $76.3(59.8-88.6)$ & $0.40(0.19-0.67)$ & $0.29(0.14-0.61)$ \\
\hline & $\mathrm{ACD} \leq 2.40 \mathrm{~mm}$ & Clinical opinion & $82.3(56.6-96.2)$ & $76.7(64.0-86.6)$ & $0.39(0.15-0.67)$ & $0.27(0.0-0.62)$ \\
\hline & $\mathrm{ACV} \leq 124 \mathrm{~mm}^{3}$ & ISGEO & $84.6(69.5-94.1)$ & $78.9(62.7-90.4)$ & $0.39(0.24-0.62)$ & $0.36(0.22-0.55)$ \\
\hline & $\mathrm{ACV} \leq 124 \mathrm{~mm}^{3}$ & Clinical opinion & $94.1(71.3-99.9)$ & $58.3(44.9-70.9)$ & $0.27(0.08-0.51)$ & $0.19(0.0-0.47)$ \\
\hline
\end{tabular}

the technique's utility in screening for angle closure. For example, Congdon et al. ${ }^{30}$ analyzed data from more than 500 subjects and concluded that the van Herick test performed less well than ultrasound biomicroscopy. Similarly, Thomas et al. ${ }^{31}$ cited both low sensitivity (61.9\%) and low specificity as the reasons for the inadequacy of van Herick for use in screening for occludable angles, despite the specificity almost reaching $90 \%$.

Figure 2 compares the current study results to sensitivity and specificity estimates from the literature for detection of narrow ACAs using the traditional van Herick threshold of grade 2 or less $(\leq 25 \%)$. These data should be interpreted with knowledge of variations in design, population demographics, and sample size between studies. Population-based crosssectional studies in East Asia found higher sensitivities but lower specificities than the present study. ${ }^{17,23}$ The report of Foster et al. ${ }^{17}$ described the use of a modified LACD grading scheme that expanded the standard van Herick grading scheme to a seven-point percentage grading scale, including subdividing grade 1 into $5 \%$ and $15 \%$ subcategories. These additional subdivisions led to improved test specificity when compared with the $\leq 25 \%$ cutoff criterion. Interestingly, mean specificities for the present study exceeded $90 \%$ for each of the LACD thresholds $\leq 25 \%, \leq 15 \%$, and $\leq 5 \%$.

Another practical consideration of the van Herick test is whether the technique should be performed at the nasal and/ or temporal limbus. Alsbirk, ${ }^{32}$ evaluating the effectiveness of the van Herick test, observed marked asymmetry in grades between the temporal and nasal aspects, with shallower LACDs temporally. This trend was common to both the Inuit and Danish populations evaluated, although not confirmed by gonioscopy. Foster et al. ${ }^{17}$ suggested that this asymmetry in temporal and nasal grades reported by Alsbirk $^{32}$ may be a reflection of variation in the position of the limbus. We observed similar van Herick grades and diagnostic performances for the detection of gonioscopically narrow angles between nasal and temporal positions, suggesting that recording of either the temporal or nasal LACD would be sufficient for casefinding in at-risk individuals.

Only one report has evaluated the diagnostic performance of Smith's test. ${ }^{33}$ Although these authors observed a good correlation between Smith's method and gonioscopy (Spearman rho $=0.938)$, this does not necessarily imply good agreement between the two techniques. Our findings revealed a lower partial AUROC for Smith's test compared with the van Herick test. Although Smith's test does not provide a diagnostic advantage over the van Herick test, there is a case for performing Smith's test when van Herick is not possible, for example in the presence of a pronounced arcus senilis. Moreover, the van Herick technique and Smith's test may be performed in rapid succession using the biomicroscope. In the present study, 100\% of narrow angle subjects defined by a gonioscopic classification based on a clinical opinion of occludability were detected based on failure of one or both tests.

In the current study, ACA was the best-performing parameter for the detection of narrow angles using the Visante OCT (based on the ISGEO classification). Wirbelauer et al. ${ }^{4}$ similarly reported a high sensitivity and specificity ( $86 \%$ and 95\%, respectively) for ACA measured with the Visante AS-OCT to detect occludable angles in a German clinic-based population. Interestingly, studies conducted in East Asia based on qualitative assessment of AS-OCT images for contact between the peripheral iris and any part of the angle wall anterior to the scleral spur, found equivalently high sensitivities, $5,34-37$ but reported specificities as low as $51 \%,{ }^{35} 55 \%,{ }^{5}$ and $58 \% .{ }^{36}$ It is unclear whether these low specificities reflect differences in definitions of narrow quadrants using AS-OCT and gonioscopy, or if angle closure is being missed by gonioscopy in these populations.

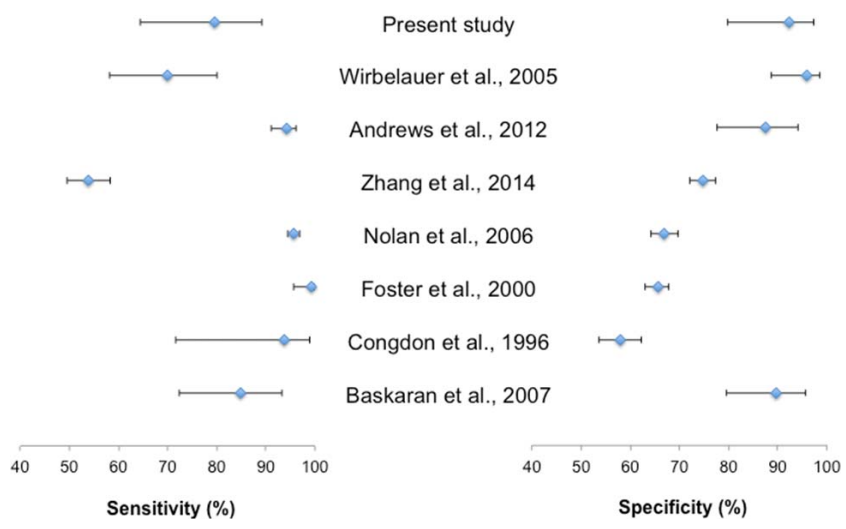

FIGURE 2. Sensitivity and specificity estimates with associated 95\% CIs for detection of narrow ACAs using the van Herick cutoff point of less than or equal to grade $2(\leq 25 \%)$. 
Pentacam imaging systems aim to overcome subjectivity associated with acquiring biometric ACA data by using fully automated analysis. In our population, Pentacam parameters showed only moderate ability to distinguish between open and narrow angles. Using the ISGEO gonioscopic definition of a narrow angle, ACA and ACD Youden cutoffs yielded sensitivity and specificity estimates between $70 \%$ and $80 \%$. These findings contrast with previous reports of Pentacam ACD being an effective indicator for the detection of gonioscopically narrow angles. ${ }^{10,11,38}$

In the context of screening for a low prevalence disease such as ACG, there is an argument to combine high test specificity, ideally above $90 \%$, with an acceptably high sensitivity so as to achieve a reasonable predictive value. Partial AUROC at $90 \%$ specificity identified the van Herick and Visante OCT ACA as the best-performing index test criteria for detection of narrow angles defined by the ISGEO classification. However, analysis of OCT images relies on examiner experience to identify features of the ACA correctly and to appropriately position the angle measurement tool. In the current study, intraobserver repeatability of ACA estimates for observations of the initial scan revealed wide 95\% CIs. Overall, the van Herick test shows great potential for use in general screening and case finding of individuals at risk of ACG. The test affords further advantage by using the biomicroscope, a standard item of equipment in ophthalmic clinics, and the van Herick test does not require any auxiliary attachments. In comparison, practitioners are less likely to invest in advanced imaging systems dedicated solely to anterior segment imaging, given the significant costs, the space taken up by the equipment, and additional training requirements to acquire and interpret images correctly.

The prevalence of narrow angles in our population was $21.8 \%$ based on the ophthalmologist's clinical opinion of occludability, compared with $53.8 \%$ using the ISGEO classification. Therefore, 25 subjects with gonioscopically narrow angles defined by ISGEO would not be eligible for prophylactic treatment, based on the ophthalmologist's clinical opinion of occludability, but periodic review for repeat goniolens assessment would observe for possible conversion to PAC/ PACG. Risks and benefits of performing prophylactic laser iridotomy on all individuals observed with gonioscopically narrow angles still need to be assessed definitively. Peripheral laser iridotomy is considered a relatively safe procedure but carries a small risk of complications, such as inflammation.

The potential for partial verification bias was addressed in this study by performing the same reference standard gonioscopic assessment on every subject. All index tests and the reference standard examination were performed on the same day, thereby addressing any risk of disease progression bias. Index-test examiners were masked to findings of other ocular examinations, including gonioscopic observations. Furthermore, index test data were interpreted independently without knowledge of the reference standard diagnosis or other test performances and vice versa. However, the study may be subject to spectrum bias, as subjects were not sampled using a population-based approach, but recruited from glaucoma and general ophthalmology clinics to form a cohort with open and narrow ACAs. Demographic data revealed a high proportion of subjects of South Asian origin, which does not represent the United Kingdom as a whole. Furthermore, it was not possible to recruit large numbers, resulting in wide CIs around diagnostic estimates of sensitivity, specificity, and partial AUROC curve. Current findings may not be generalizable to the UK general population, and would translate less well to East Asian populations in which prevalences and mechanisms of ACG differ. The examiners' knowledge of the higher prevalence of gonioscopically narrow angles in this enriched cohort compared with the general population may have biased observations toward more occludable angles. Further overestimation of diagnostic accuracy may have resulted from the use of present study data to derive the optimal cutoff value (using the Youden index) for tests in which there was no clinical consensus. However, the magnitude of the bias in sensitivity and specificity using datadriven selection of optimal cutoff values based on modeling has been estimated to be in the region of 5\% in studies with a sample size of 40 , with bias reducing with increasing sample size. ${ }^{39}$ It is also possible that exclusion of angle images captured in the vertical meridian using the Pentacam and Visante OCT may have influenced diagnostic results, in view of the higher prevalence of gonioscopically narrow quadrants observed superiorly both in this study, and previously. 8,40 Furthermore, Visante OCT and Pentacam ACA estimates were based on observations of a single cross-section between temporal and nasal angle positions. As variations in angle morphology can be observed within a sector, it is not known whether test performance for detecting narrow angles would have differed using data from multiple meridional cross sections.

In summary, this study provides data on the effectiveness of various noncontact methods to detect at-risk individuals, which may be used to develop case-finding or screening strategies to prevent ACG. Overall, the van Herick test and Visante OCT ACA showed best performance for detection of narrow angles both alone, and in combination. The van Herick test affords a number of advantages over Visante OCT imaging, but with continuing advances in OCT imaging, supported by advanced analytical tools, it is anticipated that this technology will play a more significant role over time.

\section{Acknowledgments}

The authors thank the United Kingdom College of Optometrists who provided a PhD studentship to Priya Dabasia to conduct this work. We are also most grateful to the clinicians who helped with data collection over the two screening days: Sonal Rughani, Bruno Fidalgo, Rinal Patel, Pooja Bij, Shima Shah, and Pak Sang Lee.

Disclosure: P.L. Dabasia, None; D.F. Edgar, None; I.E. Murdoch, None; J.G. Lawrenson, None

\section{References}

1. World Health Organization. Vision 2020 the right to sight, global initiative for the elimination of avoidable blindness; Action plan 2006-2011. Available at: http://www.who.int/ blindness/Vision2020_report.pdf. Accessed December 13, 2014.

2. Van Herick W, Shaffer RN, Schwartz A. Estimation of width of angle of anterior chamber. Incidence and significance of the narrow angle. Am J Ophthalmol. 1969;68:626-629.

3. Smith RJ. A new method of estimating the depth of the anterior chamber. Br J Ophthalmol. 1979;63:215-220.

4. Wirbelauer C, Karandish A, Haberle H, Pham DT. Noncontact goniometry with optical coherence tomography. Arch Ophthalmol. 2005;123:179-185.

5. Nolan WP, See JL, Chew PT, et al. Detection of primary angle closure using anterior segment optical coherence tomography in Asian eyes. Ophthalmology. 2007;114:33-39.

6. Park SB, Sung KR, Kang SY, Jo JW, Lee KS, Kook MS. Assessment of narrow angles by gonioscopy, Van Herick method and anterior segment optical coherence tomography. Jpn J Ophthalmol. 2011;55:343-350.

7. Pekmezci M, Porco TC, Lin SC. Anterior segment optical coherence tomography as a screening tool for the assessment 
of the anterior segment angle. Ophthalmic Surg Lasers Imaging. 2009;40:389-398.

8. Sakata LM, Lavanya R, Friedman DS, et al. Comparison of gonioscopy and anterior segment ocular coherence tomography in detecting angle closure in different quadrants of the anterior chamber angle. Ophthalmology. 2008;115:769-774.

9. Narayanaswamy A, Sakata LM, He MG, et al. Diagnostic performance of anterior chamber angle measurements for detecting eyes with narrow angles: an anterior segment OCT study. Arch Ophthalmol. 2010;128:1321-1327.

10. Hong S, Yi JH, Kang SY, Seong GJ, Kim CY. Detection of occludable angles with the Pentacam and the anterior segment optical coherence tomography. Yonsei Med J. 2009;50:525528.

11. Kurita N, Mayama C, Tomidokoro A, Aihara M, Araie M. Potential of the Pentacam in screening for primary angle closure and primary angle closure suspect. J Glaucoma. 2009; 18:506-512.

12. Grewal DS, Brar GS, Jain R, Grewal SP. Comparison of Scheimpflug imaging and spectral domain anterior segment optical coherence tomography for detection of narrow anterior chamber angles. Eye (Lond). 2011;25:603-611.

13. Rossi GC, Scudeller L, Delfino A, et al. Pentacam sensitivity and specificity in detecting occludable angles. Eur $J$ Ophthalmol. 2012;22:701-708.

14. Pakravan M, Sharifipour F, Yazdani S, Koohestani N, Yaseri M. Scheimpflug imaging criteria for identifying eyes at high risk of acute angle closure. J Ophthalmic Vis Res. 2012;7:111-117.

15. He M, Foster PJ, Johnson GJ, Khaw PT. Angle-closure glaucoma in East Asian and European people. Different diseases? Eye (Lond). 2006;20:3-12.

16. Bossuyt PM, Reitsma JB, Bruns DE, et al. The STARD statement for reporting studies of diagnostic accuracy: explanation and elaboration. Ann Intern Med. 2003;138:W1-W12.

17. Foster PJ, Devereux JG, Alsbirk PH, et al. Detection of gonioscopically occludable angles and primary angle closure glaucoma by estimation of limbal chamber depth in Asians: modified grading scheme. BrJ Ophthalmol. 2000;84:186-192.

18. Barrett BT, McGraw PV, Murray LA, Murgatroyd P. Anterior chamber depth measurement in clinical practice. Optom Vis Sci. 1996;73:482-486.

19. Jain R, Grewal SPS. Pentacam: principle and clinical applications. Journal of Current Glaucoma Practice. 2009;3:20-32.

20. Penner V. Use of the Visante for anterior segment ocular coherence tomography. Techniques in Ophthalmology. 2007; 5:67-77.

21. Devereux JG, Foster PJ, Baasanhu J, et al. Anterior chamber depth measurement as a screening tool for primary angleclosure glaucoma in an East Asian population. Arch Ophthalmol. 2000;118:257-263.

22. He M, Huang W, Friedman DS, Wu C, Zheng Y, Foster PJ. Slit lamp-simulated oblique flashlight test in the detection of narrow angles in Chinese eyes: the Liwan eye study. Invest Ophthalmol Vis Sci. 2007;48:5459-5463.

23. Nolan WP, Aung T, Machin D, et al. Detection of narrow angles and established angle closure in Chinese residents of Singapore: potential screening tests. Am J Ophthalmol. 2006;141:896-901.

24. Vargas E, Drance SM. Anterior chamber depth in angle-closure glaucoma. Clinical methods of depth determination in people with and without the disease. Arch Ophthalmol. 1973;90: 438-439.

25. Flahault A, Cadilhac M, Thomas G. Sample size calculation should be performed for design accuracy in diagnostic test studies. J Clin Epidemiol. 2005;58:859-862.

26. Youden WJ. Index for rating diagnostic tests. Cancer. 1950;3: 32-35.

27. Hillis SL, Metz CE. An analytic expression for the binormal partial area under the ROC curve. Acad Radiol. 2012;19: 1491-1498.

28. Andrews J, Chang DS, Jiang Y, et al. Comparing approaches to screening for angle closure in older Chinese adults. Eye (Lond). 2012;26:96-100.

29. Baskaran M, Oen FT, Chan YH, et al. Comparison of the scanning peripheral anterior chamber depth analyzer and the modified van Herick grading system in the assessment of angle closure. Ophthalmology. 2007;114:501-506.

30. Congdon NG, Quigley HA, Hung PT, Wang TH, Ho TC. Screening techniques for angle-closure glaucoma in rural Taiwan. Acta Ophthalmol Scand. 1996;74:113-119.

31. Thomas R, George T, Braganza A, Muliyil J. The flashlight test and van Herick's test are poor predictors for occludable angles. Aust N Z J Ophthalmol. 1996;24:251-256.

32. Alsbirk PH. Limbal and axial chamber depth variations. A population study in Eskimos. Acta Ophthalmol (Copenh). 1986;64:593-600

33. Al-Mubrad TM, Ogbuehi KC. Smith-method assessment of anterior chamber depth for screening for narrow anterior chamber angles. Indian J Ophthalmol. 2006;54:165-168.

34. Lavanya R, Foster PJ, Sakata LM, et al. Screening for narrow angles in the Singapore population: evaluation of new noncontact screening methods. Ophthalmology. 2008;115: $1720-1727$.

35. Sakata LM, Wong TT, Wong HT, et al. Comparison of Visante and slit-lamp anterior segment optical coherence tomography in imaging the anterior chamber angle. Eye (Lond). 2010;24: 578-587.

36. Wong HT, Chua JL, Sakata LM, Wong MH, Aung HT, Aung T. Comparison of slitlamp optical coherence tomography and scanning peripheral anterior chamber depth analyzer to evaluate angle closure in Asian eyes. Arch Ophthalmol. 2009; 127:599-603.

37. Chang DS, Sakata LM, Aung T, et al. Single versus sequential testing with scanning peripheral anterior chamber depth analyser, IOLMaster and anterior segment optical coherence tomography for the detection of narrow angles. $\mathrm{Br} \mathrm{J}$ Ophthalmol. 2011;95:1410-1414.

38. Alonso RS, Ambrosio Junior R, Paranhos Junior A, Sakata LM, Ventura MP. Glaucoma anterior chamber morphometry based on optical Scheimpflug images. Arq Bras Oftalmol. 2010;73: 497-500

39. Leeflang MM, Moons KG, Reitsma JB, Zwinderman AH. Bias in sensitivity and specificity caused by data-driven selection of optimal cutoff values: mechanisms, magnitude, and solutions. Clin Chem. 2008;54:729-737.

40. He M, Foster PJ, Ge J, et al. Gonioscopy in adult Chinese: the Liwan Eye Study. Invest Ophthalmol Vis Sci. 2006;47:47724779 . 